\title{
Vehículos Eléctricos Versus Convencionales en Colombia: Un Análisis Financiero Comparando Los Costos Totales de Propiedad
}

Torres-Pamplona, María I; Jaramillo-Duque, Alvaro ${ }^{1\left(^{*}\right)}$; Ortiz-Castrillón, José $\mathbf{R}^{1}$

${ }^{1}$ Universidad de Antioquia, Facultad de Ingeniería, Medellin, Colombia.

\section{RESUMEN}

Los vehículos eléctricos ganan cada vez más aceptación y se consideran una solución interesante de movilidad sostenible y de bajas emisiones, pero el costo de compra es la principal barrera para su adopción masiva. En este trabajo se presenta un modelo de Costo Total de Propiedad (CTP) para automóviles de uso personal en Colombia. El objetivo es comparar un Vehículo Eléctrico (VE) y un Vehículo Convencional (VC) con características semejantes. Todos los costos que se dan durante la vida útil esperada del vehículo son considerados y analizados para los dos casos, entre ellos: costo de compra, impuesto, Seguro Obligatorio Contra Accidentes de Tránsito (SOAT), costos de mantenimiento y refacciones, revisiones técnicas periódicas, y costo de combustible o electricidad. EI CTP de ambos vehículos se calcula para una vida útil de 10 años; se utiliza el Índice de Precios al Consumidor (IPC) como la tasa de incremento anual en los costos a calcular; luego, se calcula el Valor Presente Neto (VPN), utilizando la tasa de Deposito a Termino Fijo (DTF), pues se considera la compra del vehículo como una inversión que el usuario va a realizar. Los resultados presentan y exponen los casos donde el VE ya es una opción financieramente más atractiva que un VC, considerando la distancia promedio recorrida al año por el usuario. Además, se estima cuál sería el costo de compra del vehículo eléctrico para que el TCP sea igual al de un vehículo convencional.

Palabras clave: Análisis financiero; vehículo convencional; vehículo eléctrico; valor presente neto; costos totales de propiedad

\section{Electrical Vehicles versus Conventional in Colombia: a Financial Analysis Comparing Ownership Total Costs}

\section{ABSTRACT}

Electric vehicles are gaining more and more acceptance and they are considered an interesting solution for sustainable mobility and low emissions, but the purchase cost is the main barrier to their mass adoption. This paper presents a Total Cost of Ownership (TCO) model for personal use cars in Colombia. The objective is to compare an Electric Vehicle (EV) and a Conventional Vehicle (CV) with similar characteristics. All costs that occur during vehicle expected useful life are considered and analyzed for both cases, including purchase cost, taxes, mandatory insurance in face of traffic accidents, maintenance and parts costs, periodic technical reviews, and fuel or electricity cost. The TCO for both vehicles is calculated for a product lifetime of 10 years; the Consumer Price Index is used as the annual rate of increase in costs to be calculated; then, the Net Present Value is calculated, using the Fixed Term Deposit rate, since vehicle purchase is considered as an investment that user is going to do. The results are presented and exposed for which cases, the electric vehicle is already a more financially attractive option than a conventional vehicle, considering the average distance traveled per year by the user. In addition, the purchase cost of $\mathrm{EV}$ is estimated so that the TCO is equal to CV.

Keywords: financial analysis; conventional vehicle; electrical vehicle; net present value; ownership total cost 


\section{INTRODUCCIÓN}

A mediados del 2020, el parque automotor en Colombia contaba con $15^{\prime} 730.334$ vehículos de los cuales el $23 \%$ son automóviles de uso particular según el Registro Único Nacional de Tránsito (RUNT) de Colombia (Registro Único Nacional de Tránsito, 2020), siendo uno de los principales factores de contaminación del aire en las ciudades (Universidad Pontificia Bolivariana \& Área Metropolitana del Valle de Aburrá, 2018). Según el Departamento Nacional de Planeación (DNP), las emisiones de gases y material particulado que emiten los Vehículo Convencional (VC) genera daños en la salud de los colombianos, afectando principalmente a personas mayores de 44 años y menores de 5 años (Departamento Nacional de Planeación, 2017).

Adicionalmente, los VC dependen del petróleo y esto tiene dos implicaciones importantes: 1) contribución a la crisis ambiental con el deterioro de la calidad del aire, incluyendo el calentamiento global debido a los gases de efecto invernadero como el $\mathrm{CO}_{2} ; 2$ ) las reservas de petróleo actuales se están agotando y los yacimientos petrolíferos que se esperan descubrir son cada vez menores, lo que conlleva a un aumento progresivo de los precios del combustible (Sociedad de Técnicos de Automoción, 2011). Las reservas de petróleo en Colombia no son una garantía para la autonomía energética en el país, pues los nuevos yacimientos sólo aseguran reservas para los próximos 6 u 8 años (Casa Editorial El Tiempo, 2020). Estas son algunas de las razones por las que en Colombia se deben buscar alternativas para migrar su parque automotor de Vehículo Convencional (VC) a Vehículo Eléctrico (VE).

Una de las iniciativas que hace parte de las metas del Plan Nacional de Desarrollo (PND) para mejorar la calidad del aire en el país consiste en la Estrategia Nacional de Movilidad Eléctrica y Sostenible (Ministerio de Ambiente y Desarrollo, 2019). Esta estrategia busca tener al menos $6.600 \mathrm{VE}$ para el $2.022 \mathrm{y}$ 600.000 para el 2.030, con el fin de progresar en cuanto a movilidad de cero y bajas emisiones. Para cumplir esta meta, el gobierno colombiano ha decretado la ley 1964 de 2019 en la cual se promueve el uso de VE en el país con beneficios como: los impuestos no podrán superar el $1 \%$ del valor comercial del vehículo, descuento del $10 \%$ en el SOAT, descuento en el valor de la Revisión Técnico-Mecánica y de emisiones de contaminantes, a nivel territorial podrá haber descuentos en el registro o impuesto vehicular, tarifas diferenciadas de parqueaderos o exenciones tributarias, exención del pico y placa y día sin carro (Presidencia de la República de Colombia, 2019).

Los potenciales compradores de VE se desaniman principalmente porque el valor de compra es muy superior a los VC, además el desconocimiento sobre los detalles del mantenimiento y los costos asociados se ha vuelto una barrera a la hora de adquirirlos. Sin embargo, el mantenimiento y consumo de energía eléctrica de un VE es más barato comparado con el mantenimiento y consumo de combustible de un VC (Sarmiento, 2015). El elevado precio de compra de los VE se atribuye principalmente a las baterías, pues siguen siendo una tecnología emergente con expectativas de mejorar mucho en los próximos años respecto al abaratamiento, producción en masa y mayor densidad energética (Ordoñez, 2011). Sumado a este problema, están las pocas opciones que ofrecen los bancos para financiar la compra de $V E$, lo que incrementa la brecha y hace más complicado adquirir un vehículo de este tipo.

Otro factor que suele desmotivar a las personas para comprar un VE es que la infraestructura de cargadores públicos apenas se está consolidando. Actualmente, en Colombia hay algunas iniciativas de empresas privadas y públicas para disponer de infraestructura de carga. Por ejemplo, la empresa Enel-Codensa tiene una línea llamada Enel $X$ en la cual ofrecen la infraestructura eléctrica y de carga para VE públicos y particulares (Enel-Codensa, 2019). En Bogotá se han instalado 57 puntos de recarga pública y 350 más en hogares y empresas. En el Valle de Aburrá, Empresas Públicas de Medellín (EPM) cuenta con 14 estaciones de carga pública para VE, además en sus modelo de negocio, ofrece opciones de carga en casas y empresas (EPM, 2018). Igualmente, la empresa Celsia cuenta con 18 puntos de recarga en Colombia (Medellín y Área Metropolita- 
na, Bogotá, Cali, Cartagena e Ibagué) y recarga en hogares, con lo cual suma 24 estaciones en todo el país (Celsia, 2020). Muchas de estas iniciativas son de origen privado y están cubriendo la creciente necesidad de estaciones de carga en las principales ciudades, lo que no representaría un gasto adicional al gobierno.

Es indudable que la migración hacia una movilidad eléctrica tiene ventajas y retos asociados. En este trabajo se pretende estimar y responder, considerando los costos para el 2020, ¿Cuál es la distancia mínima que debe recorrer el dueño de un VE para que los costos totales de poseer éste sean semejantes o los de un VC?. Para los usuarios de vehículos que no lleguen a esa distancia mínima, se espera estimar, basado en los datos actuales ¿Cuánto tiene que bajar el precio de adquisición de los VE para que los costos totales de poseer éstos sean semejantes o a los de un VC?. Por lo tanto, este artículo presenta un análisis técnico-económico de Costos Totales de Poseer (CTP) un VE y un VC durante su vida útil; se da una estimación sobre cuándo un VE es mejor inversión que un VC, según los kilómetros que recorre el usuario al año y el costo de compra de un VE; el análisis se realiza utilizando el Valor Presente Neto (VPN), con el fin de comparar financieramente las dos alternativas.

Este artículo presenta el análisis financiero mediante las siguientes secciones: 1) en la introducción se presenta el contexto colombiano respecto al parque automotor y la problemática relacionada con la migración a una movilidad eléctrica y sostenible. 2) En la sección 2, se presenta la metodología a utilizar con el VPN y las estimaciones para el cálculo de los costos variables de los vehículos a analizar; 3) En la sección 3 , se presenta un caso de estudio comparando uno de los VC más comerciales en Colombia (Renault Sandero) y un VE con características similares de la misma marca (Renault Zoe). 4) El análisis y discusión de los resultados se presentan en la sección 4, donde también se exponen los retos futuros asociados a movilidad eléctrica en el contexto colombiano. 5) Finalmente, se presentan las conclusiones más relevantes.

\section{DESCRIPCIÓN DE LA METODOLOGÍA A UTILIZAR}

A continuación, se presenta un análisis financiero y el procedimiento a seguir para comparar los costos totales de propiedad utilizando el concepto de VPN. Comparar los costos totales de propiedad de ambos vehículos (VE vs VC) durante su vida útil, permitirá valorar el costo real e indicar cual es la mejor alternativa. Para esta evaluación, se tuvieron en cuenta las siguientes consideraciones: 1) los incentivos dados por el gobierno que reducen impuestos y dan descuentos para la compra y mantenimiento de los vehículos eléctricos; 2 ) el costo de mantenimiento de los diferentes tipos de vehículos y 3) El costo del combustible y de la electricidad.

\section{Valor Presente Neto (VPN)}

El VPN es un método que se aplica para comparar proyectos financieramente. Este método se utiliza, para tomar decisiones al momento de invertir en un proyecto que tendrá flujos de efectivo en el tiempo (Bu, 1981). El propósito en este caso es determinar los costos totales de propiedad de ambos vehículos de forma individual y llevar estos costos a un mismo tiempo. Usualmente se llevan todos los costos a un tiempo cero, para este caso, será el tiempo de compra del vehículo. De esta forma los dos proyectos son comparables incluso si los flujos de efectivo son en periodos o tiempos diferentes.

Este método permite comparar financieramente, cuál de las dos opciones es mejor (en este caso la compra de un vehículo eléctrico o de combustión), en la ventana de tiempo analizada, estimando los costos anuales. Para esto se utiliza la ecuación (1); donde $n$ es el número de años a evaluar, $C_{t}$ es el costo total del vehículo durante el año $t$, el $D T F=4.43 \%$ es la tasa de incremento anual a utilizar y $C_{C}$ es el costo de compra del vehículo.

$$
V P N=\sum_{t=1}^{n} \frac{C_{t}}{(1+D T F)^{t}}+C_{c}
$$

Los costos totales por año $\left(C_{t}=C_{f t}+C_{v t}\right)$ están divididos en dos partes, los costos fijos $\left(C_{f t}\right)$ y los 
costos variables $\left(C_{v t}\right)$. Los costos fijos anuales $\left(C_{f t}=I R_{t}+R T M G_{t}+S O A T_{t}\right)$ están dados por el Impuesto al Rodamiento $\left(I R_{t}\right)$, Revisión Tecno-Mecánica y de Gases $\left(R T M G_{t}\right)$ y el Seguro Obligatorio contra Accidentes de Tránsito ( $S O A T_{t}$ ); estos costos son obligatorios y se deben asumir cada año para garantizar la seguridad y la libre circulación del vehículo en el territorio colombiano. Los costos variables $\left(C_{v t}=C_{M T O t}+C_{C O M t}\right)$ anuales están dados por el mantenimiento $\left(C_{M T O t}\right)$, y el combustible $\left(C_{\text {COMt }}\right)$ que en general dependen del uso del vehículo (kilómetros recorridos en el año $t$ ); básicamente, entre más se use el vehículo mayor será su consumo de combustible o electricidad así como su desgaste y los costos de mantenimiento, como reparaciones y cambio de llantas entre otros.

Para evaluar los costos de mantenimiento de un vehículo convencional en el año $t$ se utiliza la ecuación (2), que se obtuvo mediante la evaluación e interpolación de los costos de mantenimiento a varios vehículos, teniendo en cuenta el Índice de Precios al Consumidor $\left(I P C_{O}=3.62 \%\right)$ en el año cero. El $C_{M T O t}$ dependerá del $I P C_{t}$ en el año $t$, de los kilómetros recorridos $\left(\mathrm{km}_{t}\right)$ y del rendimiento del combustible $\left(\mathrm{km}_{\text {galón }}\right)$ que tiene cada vehículo en sus características. La ecuación (3) presenta los costos por consumo de combustible $\left(C_{\text {COMt }}\right)$ donde el costo de combustible base $\left(C_{\text {Cbase }}\right)$ es el costo del combustible en el año inicial, para este caso la gasolina.

$$
\begin{aligned}
& C_{\text {MTOt }}=k m_{t}\left(k m_{\text {galón }}+5 t\right)\left(1+I P C_{t}\right)^{t} \\
& C_{\text {COM } t}=C_{\text {Cbase }}\left(\frac{k m_{t}}{k m_{\text {galón }}}\right)\left(1+2 I P C_{t}\right)^{t}
\end{aligned}
$$

De la misma manera, las ecuaciones 4 y 5 presentan los costos de mantenimiento y consumo de energía de un vehículo eléctrico. Estas ecuaciones tienen en cuenta los descuentos dados por el gobierno y el costo del kWh $\left(C_{k W h}\right)$.

$$
\begin{gathered}
C_{\text {MTOt }}=100 * k m_{t}\left(\frac{k W h}{k m}\right)\left(1+I P C_{t}\right)^{t} \\
C_{\text {COM } t}=k m_{t} C_{k w h}\left(\frac{k W h}{k m}\right)\left(1+I P C_{t}\right)^{t}
\end{gathered}
$$

\section{RESULTADOS}

Esta sección presenta los resultados de calcular los costos totales de poseer un Renault Sandero (VC y un Renault Zoe (VE) para una vida útil de 10 años, sin considerar seguros a terceros y/o de robo y tomando los precios promedio de 8.150 COP para el galón de gasolina y $600 \mathrm{COP}$ el kWh. Se eligieron estos dos vehículos ya que están disponibles en el mercado colombiano y tienen características semejantes en capacidad de pasajeros y equipaje, así mismo con elementos de seguridad y confort.

En la tabla 1 se presentan las características principales de los vehículos a comparar donde se pueden hacer 3 observaciones importantes: 1) el costo de compra del Zoe es aproximadamente el triple que el costo del Sandero; 2) el costo de desplazamiento por kilómetro del VE corresponde a menos de la mitad que el costo del VC; 3 ) el costo de mantenimiento del primer año del VE también corresponde a menos de

\begin{tabular}{|c|c|c|}
\hline Vehículo & Sandero (VC) & Zoe (VE) \\
\hline Tipo & Combustión & Eléctrico \\
\hline Marca & Renault & Renault \\
\hline Referencia & Life & Life \\
\hline $\begin{array}{l}\text { Emisiones contaminantes } \\
\text { (ciclo mixto WLTP) }\end{array}$ & $115-141 \mathrm{~g} / \mathrm{km}$ & $0 \mathrm{~g} / \mathrm{km}$ \\
\hline Costo de compra (2020) & $42 ’ 300.000 \mathrm{COP}$ & $107^{\prime} 500.000 \mathrm{COP}$ \\
\hline $\begin{array}{l}\text { Rendimiento (ciclo mixto } \\
\text { ciudad-carretera) }\end{array}$ & 40 km/galón & $\begin{array}{c}7.4 \mathrm{~km} / \mathrm{kWh}-136 \\
\mathrm{Wh} / \mathrm{km}\end{array}$ \\
\hline $\begin{array}{l}\text { Rendimiento (ciclo mixto } \\
\text { ciudad-carretera) }\end{array}$ & 40 km/galón & $\begin{array}{c}7.4 \mathrm{~km} / \mathrm{kWh}-136 \\
\mathrm{Wh} / \mathrm{km}\end{array}$ \\
\hline Costo desplazamiento & $203,4 \mathrm{COP} / \mathrm{km}$ & $85,68 \mathrm{COP} / \mathrm{km}$ \\
\hline $\begin{array}{l}\text { Costo mantenimiento año } \\
1(10.000 \mathrm{~km} / \mathbf{a n ̃ o})\end{array}$ & 466.290 COP & $145.068 \mathrm{COP}$ \\
\hline
\end{tabular}
la mitad del costo del VC.

Tabla 1. Características principales de los vehículos a evaluar.

\subsection{Costos Totales dependiendo de los km/año reco-} rridos

La Figura 1 muestra los costos totales de poseer un VC y un VE (curva azul y roja respectivamente) en función de la distancia promedio anual recorrida, desde $10.000 \mathrm{~km}$ hasta $60.000 \mathrm{~km}$; se puede observar que la curva azul, VC, tiene una pendiente mayor que la curva roja, VE. Si el usuario recorre aproximadamente $33.000 \mathrm{~km}$ al año (intersección de las dos curvas) o más, entonces el vehículo eléctrico es una mejor opción desde un punto de vista financiero, a 
pesar del elevado costo de compra del VE. En la intersección de las curvas, los costos totales de poseer un vehículo serán aproximadamente iguales, sin embargo, el vehículo eléctrico tendrá ventajas adicionales como la excepción del pico y placa, reducción en la contaminación auditiva y la cero emisión de gases contaminantes. Si el dueño del VE recorre más de $33.000 \mathrm{~km}$ al año, los costos totales de poseer un vehículo serán menores a los de un VC.

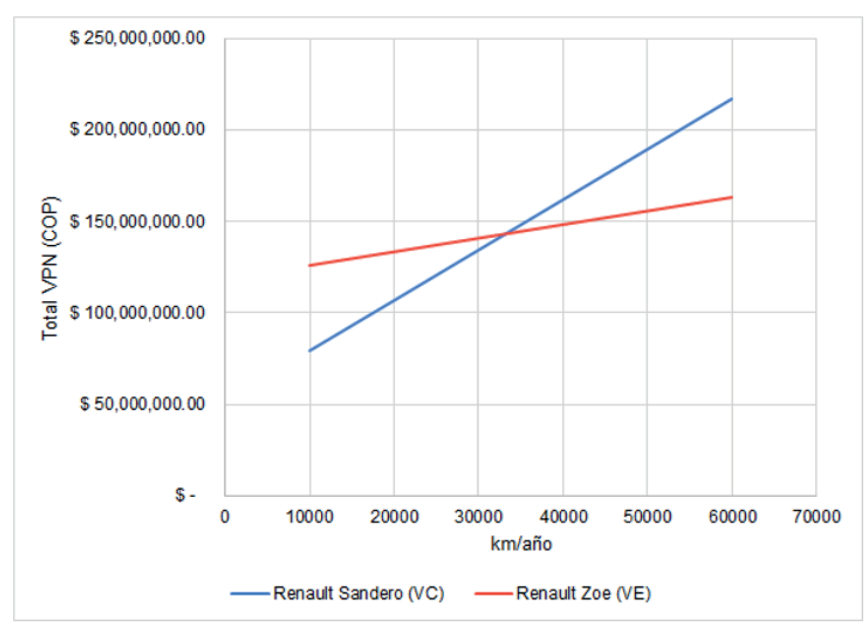

Figura 1. VPN vehículo convencional vs vehículo eléctrico.

\subsection{Costos totales dependiendo del precio de compra del VE}

La Tabla 2 presenta los resultados obtenidos, mediante un proceso de optimización utilizando el Solver de Excel, donde se buscaba estimar el precio futuro del VE, tal que los costos totales de poseer ambos vehículos fueran iguales para una misma distancia recorrida al año. En los resultados se puede observar que para una persona que recorre 33.000 $\mathrm{km} / \mathrm{año} \mathrm{y} \mathrm{con} \mathrm{el} \mathrm{precio} \mathrm{de} \mathrm{compra} \mathrm{actual} \mathrm{para} \mathrm{el} \mathrm{VE}$ de $107^{\prime} 500.000$ COP, los costos totales de poseer un vehículo son aproximadamente iguales. En contras-

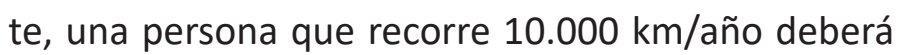
esperar a que el precio del VE baje aproximadamente a $63^{\prime} 000.000$ COP para poder igualar los costos totales de ambos vehículos. En la tabla se presentan los resultados para diferentes distancias recorridas al año, de esta forma, la mayoría de conductores podrán estimar cuál será el valor ideal para el VE sin que signifique un aumento en los costos totales durante la ventana de tiempo de 10 años.

\begin{tabular}{lcc}
\hline $\mathbf{k m} / \mathbf{a n ̃ o}$ & $\begin{array}{c}\text { Costo total de poseer VC } \\
\text { y VE (COP) }\end{array}$ & $\begin{array}{c}\text { Precio del Vehículo Eléctrico } \\
\text { a futuro (COP) }\end{array}$ \\
\hline 5.000 & $6^{\prime} 5^{\prime} 836.672$ & $53^{\prime} 426.655$ \\
10.000 & $7^{\prime}, 602.430$ & $63^{\prime} 007.291$ \\
15.000 & $9^{\prime} 368.187$ & $72^{\prime} 587.936$ \\
20.000 & $107^{\prime} 133.945$ & $82^{\prime} 168.573$ \\
25.000 & $120^{\prime}, 999.702$ & $91^{\prime} 749.174$ \\
30.000 & $134^{\prime} 665.460$ & $101^{\prime} 329.815$ \\
33.000 & $142^{\prime} 924.915$ & $107^{\prime} 078.233$ \\
\hline
\end{tabular}

\section{ANÁLISIS DE RESULTADOS, DISCUSIÓN Y RETOS FUTUROS}

Los resultados obtenidos de calcular los costos totales dependiendo de la distancia recorrida al año por el usuario, muestran dos puntos importantes a tratar, considerando solo el aspecto económico: 1) EI primero indica que para un usuario que recorre más de 33.000 km/año, el VE es una mejor opción pues los costos totales, asumiendo 10 años para la evaluación, son menores comparada con el VC. Por lo tanto, los usuarios a quienes favorece invertir en un VE son aquellos que usan su vehículo de forma continua, por ejemplo, asesores de ventas, consultores a domicilio o plataformas de transporte (uber, cabify, Didi, inDrive, etc); estos usuarios utilizan el vehículo la mayor parte del día y por tal razón llegan y sobrepasan promedios superiores de 33.000 km/año. Considerando el caso específico de los taxis, estos suelen recorrer distancias superiores a los 33.000 km/año, por lo que su viabilidad financiera está garantizada utilizando un vehículo eléctrico. 2) Actualmente, el uso típico de vehículos en Colombia de usuarios particulares, está entre 8.000 y 15.000 km/año (dependiendo de la ciudad); esto significa que, con los costos actuales, el VE aún no es una opción financieramente viable para usuarios particulares.

Los resultados obtenidos de calcular los costos totales dependiendo del precio de compra del VE coinciden con los $33.000 \mathrm{~km} / \mathrm{año}$. Si el usuario recorre más

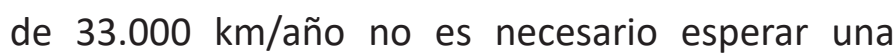
reducción del precio de compra del VE, ya que es una mejor inversión, en este punto los costos totales son iguales para ambos vehículos. Un usuario particular que use su vehículo entre 8.000 y 15.000 km/año, deberá esperar a que el precio de compra se reduzca hasta quedar entre $60^{\prime} 000.000$ y $70^{\prime} 000.000$ aproximadamente.

Estudios realizados respecto a la reducción en el 
precio de compra de los VE muestran una disminución gradual debido a la economía de escala, al desarrollo de la tecnologías involucradas y abaratamiento en los procesos de producción entre los principales factores. Las estimaciones varían dependiendo del segmento del vehículo, los vehículos de gama alta ya podrían estar llegando incluso a la paridad del costo inicial, sin embargo, para los vehículos de gama media y baja, se estima que, para antes del 2030, se de igualdad en los costos totales o incluso del costo inicial (van Velzen et al., 2019) (Soulopoulos, 2017).

Adicionalmente, en el mercado automotriz ya se está dando el efecto Osborne, lo que significa que los potenciales compradores de vehículos están a la espera que la demanda de VE aumente y se abarate, lo que implica una disminución en la demanda de VC. (Maarten Vinkhuyzen, 2019). Este efecto ya obliga al sector automotriz a acelerar su transición $y$, por ende, a ofrecer más opciones de vehículos eléctricos, de lo contrario sus ingresos se verán afectados (Pérez, 2020). Las estimaciones sobre el abaratamiento de los vehículos eléctricos tienen diferentes niveles de optimismo, sin embargo la mayoría de las estimaciones concuerdan con una constante reducción en las baterías y, con esta, de los vehículos, debido a los nuevos desarrollos y a la economía de escala (Henze, 2019), (Holland, 2019).

La empresa Tesla Inc. que diseña, fabrica y vende vehículos eléctricos, viene realizando un evento anual Ilamada "Battery Day", en su versión del 2020, realizado el pasado 22 de septiembre, anunció que reducirían sus costos actuales de producción de las batería en un $54 \%$, a la vez que aumentarán la densidad energética en un 56\% (Tesla, 2020). Estos avances impactarán los mercados en los próximos años y fomentará la competencia entre las empresas del sector.

\subsection{Incentivos gubernamentales y problemática de salud pública}

Algunos países ofrecen ayudas para los compradores de VE, por ejemplo en China, Alemania y USA se otorgan entre 2,400 hasta 7,500 Euros en subsidios para la compra de un VE nuevo; en Noruega los VE no están sujetos a impuestos de importación ni al IVA y se otorgan descuentos en las tarifas de peajes y estacionamiento; Francia otorga bonos ecológicos por 6.000 euros para la compra de VE, además ofrece 2.500 euros por llevar al desguace el VC viejo; en México la tarifa de la energía eléctrica, destinada para la carga de VE tienen un descuento del 40\%; finalmente, en Ecuador todos los VE están exentos de aranceles e impuestos (Volkswagen, 2019),(SEGOB, 2015), (Münzel et al., 2019), (Wang et al., 2019).

Si bien, Colombia está incursionando en el campo de la movilidad eléctrica, los incentivos actuales no son suficientes. Además las metas propuestas en el Plan Nacional de Desarrollo (PND) para la Estrategia Nacional de Movilidad Eléctrica y Sostenible, no significarán un cambio sustancial en la proporción de VE respecto al total de VC (Ministerio de Ambiente y Desarrollo, 2019); ya que se espera tener al aproximadamente 6.600 VE para el 2.022 y 600.000 para el 2.030 , lo que sería un $0.04 \%$ y $3.9 \%$ respectivamente, del total de vehículos registrados al 2020 en el RUNT.

La combustión de los VC genera gases y compuestos químicos que quedan suspendidos en el aire como dióxido de carbono $\mathrm{CO} 2$, hidrocarburos no quemados $\mathrm{HC}$, monóxido de carbono $\mathrm{CO}$, óxido de nitrógeno NOx, dióxido de azufre SO2 y Compuestos Orgánicos Volátiles (COV) (IDEAM, s. f.). Estos generan un inminente riesgo en la salud de las personas si se tiene una exposición a ellos de forma frecuente. Además, los VC generan material particulado PM10 y PM2.5, la primera se queda retenida en las vías respiratorias generando daños en el sistema respiratorio, la segunda tiene la capacidad de atravesar tejidos y llegar al torrente sanguíneo causando daños en cualquier órgano o sistema (Instituto para la Salud Geoambiental, 2013).

Está problemática se puede reducir con la migración del parque automotriz convencional a VE debido a que estos no requieren combustión en un motor, por lo tanto, no generan dichas partículas contaminantes, lo que disminuye la contaminación del aire, beneficiando al medio ambiente y la salud de las personas. Con esto se incurre en ahorros a nivel de salud, pues en Colombia la contaminación del aire es generadora de altos costos sociales, estimándose en aproximadamente 15,4 billones COP anuales en relación a salud pública, mortalidad y movilidad; lo que equivale al 1,93\% del PIB de 2015 (Departamen- 
to Nacional de Planeación, 2017). Esto quiere decir que la migración de VC a VE favorece a la reducción de muertes prematuras por enfermedades respiratorias, y a la reducción de costos de hospitalizaciones, consulta externa y urgencias. (Muñoz, 2012).

Un ejemplo de este fenómeno se puede apreciar en el estudio "Clearing The Air" (Limpiando el aire) realizado por el organismo canadiense "Environmental Defence" (Environmental Defence, 2020) y la Asociación de Salud Pública de Ontario (Ontario Public Health Association, 2020). En el estudio se analizó el área metropolitana de Toronto, donde se consideró que todos los automóviles y camionetas fueran eléctricos; con este cambio, se estimó que se podrían evitar 313 muertes prematuras al año y un beneficio social estimado de $\$ 2.4$ mil millones de dólares en ahorros al sistema sanitario de la región (Berman, 2020).

Considerando los costos que se podrían evitar al sistema de salud colombiano al migrar de forma masiva a VE, se podría diseñar un plan de promoción más ambicioso donde se eliminen por completo el IVA, se fomente de forma económica la chatarrización de VC antiguos y se otorguen subsidios para la compra de VE nuevos. Por otro lado, las entidades bancarias deberían recibir beneficios o incentivos, si ofrecen productos financieros con tasas especiales para la adquisición exclusiva de VE y de esta forma facilitar y acelerar la transición hacia una movilidad más sostenible en Colombia.

\subsection{Costos para adecuar la infraestructura de carga en Colombia}

Para Europa, fue realizando un estudio por la organización "Transport \& Environment", donde se estimó que la inversión adicional necesaria para garantizar una cobertura adecuada de estaciones de carga para viajes en todo el territorio europeo, supondría un aumento del $1 \%$ en el 2020, un 3\% para el 2025 y un $5 \%$ para el 2030 del presupuesto asignado al mantenimiento de la infraestructura vial. Adicionalmente, si se destinaran los subsidios que la Unión Europea hace anualmente para la industria de hidrocarburos, aproximadamente en un año y medio, se podría cubrir el total de la inversión necesaria proyectada hasta el 2030 (Transport \& Environment, 2020).

Es claro que la infraestructura eléctrica debe ser adecuada para garantizar los suficientes puntos de carga públicos y privados (Dinero, 2019); por lo tanto, los operadores de las redes de distribución ya están realizando planes de adecuamiento, además, sumado a esto el sector eléctrico está pasando por una transición hacia sistemas más confiables, sostenibles y resilientes, donde la movilidad eléctrica es un aspecto muy importante (Colombia Inteligente, 2016).

Es posible que en Colombia la necesidad de mejorar la infraestructura eléctrica implique una inversión significativa, sin embargo, estas acciones son progresivas y generarán ganancias derivadas de su uso. Si se diseña una adecuada regulación y modelo de negocio, será posible que las empresas vean una opción de aumentar sus ganancias con las estaciones de carga públicas y que no implique una inversión significativa del gobierno.

\section{CONCLUSIONES}

En este artículo se realizó un análisis financiero donde se comparan los costos totales de poseer un VC y un VE. Para esto, se llevaron todos los flujos de efectivo utilizando el concepto de VPN en una ventana de tiempo de 10 años a partir del 2020. De manera general, se calcularon los costos fijos y variables para ambos vehículos teniendo en cuenta las tasas de variación en los precios DTF e IPC. Además, se consideraron los incentivos que el gobierno colombiano otorga actualmente para los VE. Los resultados muestran que para un usuario que recorre más de $33.000 \mathrm{~km}$ al año, el VE es una mejor opción desde un punto de vista financiero, pues los costos totales de poseer un VC serán mayores que los de un VE. Actualmente, el recorrido promedio de un usuario en una ciudad está entre 8.000 y 15.000 $\mathrm{km}$ al año, lo que hace que la compra de un VE no sea una opción económicamente viable para un usuario particular; en consecuencia, el usuario particular deberá esperar a que el costo de compra del VE baje y/o que los incentivos del gobierno aumenten para hacer del VE una opción financieramente viable. Los usuarios que superan los $33.000 \mathrm{~km}$ al año pueden optar por un VE ya que, a partir de este recorrido mínimo, y a pesar del elevado costo de compra, el VE es una mejor opción financiera considerando una vida útil del vehículo de 10 años. 
En los próximos años se espera que el costo inicial de los vehículos eléctricos continúe decreciendo, implicando que los costos totales de poseer VE sean aproximadamente iguales o inferiores en la mayoría de los segmentos, comenzando por los vehículos de gama alta y hasta llegar a los vehículos de gama baja o más baratos. Este fenómeno ya se está dando y la competencia entre los principales constructores de vehículos es una garantía de que los precios continuarán bajando.

Uno de los principales requisitos para la implementación masiva de los VE es la infraestructura pública de carga. Al día de hoy, no hay una cobertura completa en el territorio colombiano, sin embargo, esta infraestructura ya se está desarrollando y aumentando la disponibilidad de estos servicios en las principales ciudades del país. Es cuestión de unos pocos años para que sea posible viajar entre las principales ciudades y disponer de puntos de carga pública que permita hacer este tipo de recorridos sin necesidad de disponer de vehículos eléctricos con baterías de gran autonomía.

La implementación masiva de los VE no solo ofrece beneficio financiero a los dueños que superen los $33.000 \mathrm{~km}$ al año. También hay que considerar el beneficio ambiental por la disminución de las emisiones, partículas y el ruido que producen los VC. Otro factor muy importante es la reducción de las muertes prematuras y los costos del tratamiento de enfermedades respiratorias. Los gobiernos deberían considerar estos beneficios y los ahorros que se podrían dar, con el fin de ofrecer más beneficios, subsidios o productos financieros especiales que promuevan la implementación masiva de VE.

La soberanía energética es un factor muy importante y no se han considerado todas las implicaciones a corto y mediano plazo. Actualmente Colombia tiene una dependencia a los hidrocarburos que, además, cada vez son más escasos y sus precios más volátiles. Esta dependencia hace que Colombia no controle completamente su cadena productiva. Sin embargo, con una migración masiva a VE y con un plan de expansión de la infraestructura eléctrica, esta dependencia se reduciría. Adicionalmente, la mayoría de los nuevos proyectos energéticos en Colombia son con fuentes renovables de energía, que tienen un menor impacto ambiental y también contribuirían a reducir las emisiones, garantizarán el suministro eléctrico y la salud de los ciudadanos. 


\section{REFERENCIAS}

Berman, B. (2020, junio 3). New study: Every electric car brings $\$ 10,000$ in life-saving benefits. Electrek. https://electrek.co/2020/06/03/newstudy-every-electric-car- brings-10000-in-life-saving-benefits/

Bu, R. C. (1981). Análisis y evaluación de proyectos de inversión. Editorial Limusa.

Casa Editorial El Tiempo. (2020, abril 30). Reservas probadas de crudo en Colombia aumentaron a 6,3 años en 2019. Portafolio.co. https://www.portafolio.co/economia/reservas-probadas-de-crudo-en-colombia-aumen taron-a-6-3-anos-en-2019-540427

Colombia Inteligente. (2016, junio 1). Plan estratégico. https://www.colombiainteligente.org/index.php/nosotros/objetivos-y-plan-estrategico

Departamento Nacional de Planeación. (2017, mayo 7). Los costos en la salud asociados a la degradación ambiental en Colombia ascienden a \$20,7 billones.

https://www.dnp.gov.co/Paginas/Los-costos-en-la-salud-asociados-a-la-degradaci\%C3 \%B3n-ambiental-en-Colombia-ascienden-a-\$20,7-billones-.aspx

Enel-Codensa. (2019, diciembre 17). Enel X ofrece infraestructura para recargar vehículos eléctricos públicos y privados. https://www.enel.com.co/content/enel-co/es/megamenu/prensa/news/2019/12/aprende-a-recargar-un-vehiculoelectrico.html

Environmental Defence. (2020). Environmental Defence. Environmental Defence.

https://environmentaldefence.ca/

EPM. (2018, febrero 1). EPM abrió nuevas Ecoestaciones en Medellín, Envigado y Sabaneta. https://www.epm.com.co/site/home/sala-de-prensa/noticias-y-novedades/epm-abrionuevas-ecoestaciones-en-medellin-envigado-y-sabaneta/

Henze, V. (2019, diciembre 3). Battery Pack Prices Fall As Market Ramps Up With Market Average At \$156/kWh In 2019. BloombergNEF. https://about.bnef.com/blog/battery-pack-prices-faII-as-maket-ramps-up-with-market-average-at-1 56-kwh-in-2019/
Holland, M. (2019, diciembre 4). Powering The EV Revolution-Battery Packs Now At \$156/kWh, 13\% Lower Than 2018, Finds BNEF. CleanTechnica. https://cleantechnica.com/2019/12/04/powering-the-ev-revoltion-battery-packs-now -at-156-kwh-13-lower-than-2018-finds-bnef/

IDEAM. (s. f.). Emisiones por fuentes móviles. Recuperado 20 de agosto de 2020. http://www.ideam.gov.co/web/contaminacion-y-calidad-ambiental/emisiones-por-fuentes -moviles

Instituto para la Salud Geoambiental. (2013, septiembre 23). Material particulado. Instituto para la Salud Geoambiental. https://www.saludgeoambiental.org/material-particulado.

Instituto para la Salud Geoambiental. (2013, septiembre 23). Material particulado. Instituto para la Salud Geoambiental.

https://www.saludgeoambiental.org/material-particulado.

Maarten Vinkhuyzen. (2019, febrero 25). The Osborne Effect On The Auto Industry. CleanTechnica. https://cleantechnica.com/2019/02/25/the-osborne-effect-on-the-auto-industry/

Ministerio de Ambiente y Desarrollo. (2019, junio 11). Colombia consolida la Estrategia Nacional de Movilidad Eléctrica | Ministerio de Ambiente y Desarrollo Sostenible. https://www.minambiente.gov.co/index.php/noticias-minambiente/4333-colombia-consolida-la-estrategia-nacio nal-de-movilidad-electrica

Münzel, C., Plötz, P., Sprei, F., \& Gnann, T. (2019). How large is the effect of financial incentives on electric vehicle sales? - A global review and European analysis. Energy Economics, 84, 104493.

https://doi.org/10.1016/j.eneco.2019.104493

Muñoz, D. C. M. (2012). Evaluación financiera y ambiental de la utilización de vehículos eléctricos en las empresas. [Tesis de grado, Universidad de San Buenaventura]. http://biblioteca.usbbog.edu.co:8080/Biblioteca/BDigital/68904.pdf

Ontario Public Health Association. (2020). Ontario Public Health Association. OPHA. http://opha.on.ca/Home.aspx 
Ordoñez, C. P. (2011). Estudio de baterías para vehículos eléctricos. [Tesis de grado, Universidad Carlos III de Madrid].

https://e-archivo.uc3m.es/bitstream/handle/10016/11805/PFC_Carlos_Pena_Ordone z.pdf? sequence $=1 \&$ isAllowed $=y$

Pérez, E. (2020, agosto 14). Todos los planes de los fabricantes para pasarse al coche eléctrico: Desde los más radicales a los que hablan de 2030. Xataka. https://www.xataka.com/automo$\mathrm{vil} / \mathrm{cuando}$-llegara-coche-electrico-estas-fechasprometidas-fabricantes-para-transicion-1

Presidencia de la República de Colombia. (2019, agosto 28). Presidente Duque lanza Estrategia Nacional de Movilidad Eléctrica y Sostenible por la calidad del aire y el transporte eficiente. https://id.presidencia.gov.co:443/Paginas/prensa/2019/Presidente-Duque-lanza-EstrategiaNacional-Movilidad-Electrica-Sostenible-calidad-aire-transporte-eficiente-190828.aspx

Registro Único Nacional de Tránsito. (2020). Parque automotor registrado en RUNT.

https://www.runt.com.co/runt-en-cifras/parque-automotor

Sarmiento, J. D. T. (2015). Estudio de viabilidad en la implementación de vehículos eléctricos en la ciudad de Cuenca [Tesis de grado, Universidad Politécnica Salesiana].

https://dspace.ups.edu.ec/bitstream/1234567 89/8050/1/UPS-CT004893.pdf

SEGOB. (2015, diciembre 24). DOF - Diario Oficial de la Federación.

http://www.dof.gob.mx/nota_detalle.php?codigo $=5421295 \&$ fecha $=24 / 12 / 2015$

Sociedad de Técnicos de Automoción. (2011).

El Vehículo Eléctrico. Desafíos tecnológicos, infraestructuras y oportunidades de negocio. Librooks.

Soulopoulos, N. (2017, abril 12). When Will Electric Vehicles be Cheaper than Conventional Vehicles? https://www.blogmotori.com/wp-content/uploads/2017/07/EV-Price-Parity-Report_ BlogMotori_COM_MobilitaSostenibile_IT.pdf
Tesla. (2020, septiembre 22). 2020 Annual Meeting of Stockholders and Battery Day | Tesla. https://www.tesla.com/2020shareholdermeeting Transport \& Environment. (2020). Draft TE Infrastructure Report Final (p. 68). https://www.transportenvironment.org/sites/te/files/publications/01\%202020\%20Draft\%20 TE\%20Infrastructure\%20Report\%20Final.pdf

Universidad Pontificia Bolivariana, \& Área Metropolitana del Valle de Aburrá. (2018). Actualización inventario de emisiones atmosféricas del valle de aburrá - año 2016 (p. 82) [Informe final]. https://www.metropol.gov.co/ambiental/calidad-del-aire/Documents/Inventario-de-emisi ones/Inventario_Fuentes M\%C3\%B3viles2016.pdf

van Velzen, A., Annema, J. A., van de Kaa, G., \& van Wee, B. (2019). Proposing a more comprehensive future total cost of ownership estimation framework for electric vehicles. Energy Policy, 129, 1034-1046.

https://doi.org/10.1016/j.enpol.2019.02.071

Volkswagen. (2019, mayo 28). How electric car incentives around the world work.

https://www.volkswagenag.com/en/news/stories/2019/05/how-electric-car-incentivesaround-the-world-work.html\#

Wang, N., Tang, L., \& Pan, H. (2019). A global comparison and assessment of incentive policy on electric vehicle promotion. Sustainable Cities and Society, 44, 597-603.

https://doi.org/10.1016/j.scs.2018.10.024 